\title{
Tauber theory for infinitely divisible variance functions
}

\author{
BENT JØRGENSEN ${ }^{1 *}$ and JOSÉ RAÚL MARTÍNEZ ${ }^{2}$ \\ ${ }^{1}$ Department of Statistics, University of British Columbia, Vancouver BC V6T 1Z2, Canada \\ ${ }^{2}$ FAMAF - Universidad Nacional de Córdoba, Ciudad Universitaria, 5000 Córdoba, Argentina
}

We study a notion of Tauber theory for infinitely divisible natural exponential families, showing that the variance function of the family is (bounded) regularly varying if and only if the canonical measure of the Lévy-Khinchine representation of the family is (bounded) regularly varying. Here a variance function $V$ is called bounded regularly varying if $V(\mu) \sim c \mu^{p}$ either at zero or infinity, with a similar definition for measures. The main tool of the proof is classical Tauber theory.

Keywords: exponential dispersion model; Lévy-Khinchine representation; Lévy measure; natural exponential family; regular variation; Tweedie family

\section{Introduction}

Consider a Radon measure $w$ on $\mathbb{R}$, define its cumulant function $k_{w}$ by

$$
k_{w}(\theta)=\log \int \exp (\theta x) w(\mathrm{~d} x)
$$

and define the interval $\Theta_{w}$ by

$$
\Theta_{w}=\left\{\theta \in \mathbb{R}: k_{w}(\theta)<\infty\right\} .
$$

Let $M(\mathbb{R})$ denote the set of non-degenerate Radon measures on $\mathbb{R}$ such that int $\Theta_{w}$ is nonempty. The natural exponential family generated by the measure $w$ in $M(\mathbb{R})$ is defined as the set of probability measures $\mathrm{P}=\left\{P_{\theta}: \theta \in \Theta_{w}\right\}$, where, for $\theta$ in $\Theta_{w}$,

$$
P_{\theta}(\mathrm{d} x)=\exp \left\{\left(\theta x-k_{w}(\theta)\right\} w(\mathrm{~d} x) .\right.
$$

The mean of $P_{\theta}$ is

$$
\mu=\tau(\theta)=\tau_{w}(\theta)=k_{w}^{\prime}(\theta),
$$

with domain $\Omega=\tau\left(\right.$ int $\left.\Theta_{w}\right)$. We define the variance function of $\mathrm{P}$ as the function $V$ : $\Omega \rightarrow \mathbb{R}_{+}$given by

$$
V(\mu)=\tau^{\prime}\left(\tau^{-1}(\mu)\right\}
$$

\footnotetext{
*To whom correspondence should be addressed. e-mail: bent@stat.ubc.ca
} 
Here $V(\mu)$ is the variance of the probability measure $P_{\tau^{-1}(\mu)}$.

The variance function and its domain, $(V, \Omega)$, characterize $\mathrm{P}$ within the class of natural exponential families. This has given rise to a number of results on the characterization of natural exponential families and their properties in terms of the variance function, an idea that goes back to Tweedie (1947), Wedderburn (1974) and Morris (1982). Various types of variance function were studied by Bar-Lev and Enis (1986), Mora (1986), Letac (1987), Bar-Lev and Bshouty (1989), Letac and Mora (1990) and Bar-Lev et al. (1991a). See Letac (1992) for a survey of recent results.

The variance function $V$ may be considered a kind of characteristic function for natural exponential familes. One reason for this is the continuity theorem of Mora (1990), by which convergence of a sequence of natural exponential families may be characterized in terms of convergence of the corresponding sequence of variance functions. This result was used by Jørgensen et al. (1994) to prove a kind of central limit theorem for natural exponential families, based on convergence of the variance function to the Tweedie form

$$
V(\mu)=\sigma^{2} \mu^{p}
$$

The Tweedie class of natural exponential families includes the gamma, Poisson and some compound Poisson models, as well as families generated from extreme stable distributions. Such models have applications in generalized linear models, cf. McCullagh and Nelder (1989) and Jørgensen (1986; 1987; 1992).

Infinite divisibility is central to the study of variance functions, because a natural exponential family with variance function $V$ is infinitely divisible if and only if $\sigma^{2} V$ is a variance function for all $\sigma^{2}>0$ (Jørgensen 1986), and because many important natural exponential families are infinitely divisible. Infinite divisibility for polynomial or powerseries variance functions was studied by Bar-lev (1987) and Bar-Lev and Bshouty (1990). For a recent survey, see Bar-Lev et al. (1991b).

The main result of the present paper (Theorem 2.4) presents a kind of Tauber theory for infinitely divisible natural exponential families. We now summarize the main results; precise definitions and results are given in Section 2.

The Lévy-Khinchine representation of an infinitely divisible measure $w$ is given by

$$
k_{w}(\theta)=\int_{\mathbb{R} \backslash\{0\}}\left\{\mathrm{e}^{\theta x}-1-\theta \gamma(x)\right\} v(\mathrm{~d} x),
$$

where $v$ is the Lévy measure and $\gamma$ a centring function. If $\mathrm{P}$ is the natural exponential family generated by $w$, the measure $P_{\theta}$ in $\mathrm{P}$ has Lévy-Khinchine representation

$$
\begin{aligned}
k_{P_{\theta}}(s) & =k_{w}(\theta+s)-k_{w}(\theta) \\
& =\int_{\mathbb{R} \backslash\{0\}}\left\{\mathrm{e}^{s x}-1-s \mathrm{e}^{-\theta x} \gamma(x)\right\} \mathrm{e}^{\theta x} v(\mathrm{~d} x),
\end{aligned}
$$

with Lévy measure $\mathrm{e}^{\theta x} v(\mathrm{~d} x)$ and centring function $\mathrm{e}^{-\theta x} \gamma(x)$. Since the new Lévy measure is an exponential tilting of $v$, many properties of the Lévy-Khinchine representation are shared by all members of the family. We shall hence refer to (1.2) simply as the Lévy-Khinchine 
representation of the natural exponential family $\mathrm{P}$, stressing differences between members of $P$ only when strictly necessary.

A variance function $V$ is said to be bounded regularly varying (at zero or infinity) if $V(\mu) \sim c \mu^{p}$ for some $0<c<\infty$. We define bounded regular variation of a measure in a similar way. A Lévy measure is said to be (bounded) regularly varying if the canonical measure $x^{2} v(\mathrm{~d} x)$ is (bounded) regularly varying.

Theorem 2.4 states that, under regularity conditions, the variance function of an infinitely divisible natural exponential family is (bounded) regularly varying if and only if the corresponding Lévy measure is (bounded) regularly varying. The proof is essentially an application of classical Tauber theory to the second derivative of the cumulant function $k_{w}$.

The main result of Jørgensen et al. (1994) is, in the present terminology, that bounded regular variation of a variance function $V$ implies that the corresponding exponential dispersion model, suitably standarized, converges to the Tweedie model corresponding to (1.1). This provides a useful corollary to Theorem 2.4.

Definitions, the main result and some comments on convergence to the Tweedie model are presented in Section 2. Section 3 contains results on regular variation, and the main proof is given in Section 4.

\section{Regular variation of Lévy measures}

This section contains basic definitions regarding the Lévy measure and regular variation, followed by the main result (Theorem 2.4).

The version of the Lévy-Khinchine representation of an infinitely divisible probability measure that we will use is adopted from Letac (1992). In particular, we use the following classification of Lévy measures. If the Lévy measure $v$ is bounded, it is said to be of type 0 . If $v$ is unbounded but

$$
\int_{\mathbb{R} \backslash\{0\}} \min (1,|x|) v(\mathrm{~d} x)<\infty,
$$

$v$ is said to be of type 1 . In all other cases, $v$ is said to be of type 2.

We now give some definitions on regular variation. Basic references for regular variation are Feller (1971, pp. 442-447), de Haan (1975) and Bingham et al. (1987).

Definition 2.1. A function $u: \mathbb{R}_{+} \rightarrow \mathbb{R}_{+}$is said to be bounded regularly varying at infinity (zero) with exponent $p \in \mathbb{R}$, if there exists a constant $c \in(0, \infty)$ such that

$$
u(x) \sim c x^{p} .
$$

Definition 2.2. A function $u: \mathbb{R}_{+} \rightarrow \mathbb{R}_{+}$is said to vary regularly at infinity with exponent $\infty$ $(-\infty)$ if there exists a function L, slowly varying at infinity, and a positive (negative) constant $c$, such that

$$
u(x)=L(x) \mathrm{e}^{c x} .
$$


If $L(\infty)=\lim _{x \rightarrow \infty} L(x)$ is positive and finite, $u$ is said to be bounded regularly varying with exponent $\infty(-\infty)$.

We note that regular variation with infinite exponent is a special case of rapid variation (de Haan 1975).

The ordinary definition of regular variation for (probability) measures is not immediately applicable to a Lévy measure $v$, because its integral does not necessarily converge to zero. Instead, we use the following definition.

Definition 2.3. Let $v$ be a Lévy measure on $(0, \infty)$, and let $\bar{M}(x)$ be the improper distribution function of the canonical measure $M(\mathrm{~d} x)=x^{2} v(\mathrm{~d} x)$, defined by

$$
\bar{M}(x)=\int_{(0, x)} t^{2} v(\mathrm{~d} t)=\int_{0}^{x} t^{2} v(\mathrm{~d} t) .
$$

If $\bar{M}$ is regularly varying (bounded regularly varying) at either zero or infinity with exponent $\beta>0$, we say that the measure $v$ is regularly varying (bounded regularly varying) with exponent $\beta-2$.

We now state the main result concerning Tauber theory for infinitely divisible natural exponential families. The following terminology will be used in the theorem. Let $(V, \Omega)$ be the variance function of a natural exponential family $\mathrm{P}$ and let $b=\inf \Omega$ and $c=\sup \Omega$. If $b>-\infty(c<\infty)$ we say that $V$ is regularly varying at $b^{+}\left(c^{-}\right)$with exponent $p \in \mathbb{R}$, if and only if $V(b+\cdot)(V(c-\cdot))$ is regularly varying at zero with exponent $p$. If $b=-\infty$, we say that $V$ is regularly varying at $-\infty$ with exponent $p \in \mathbb{R} \cup\{+\infty\} \cup\{-\infty\}$, if and only if $V(-\cdot)$ is regularly varying at infinity with exponent $p$.

In the theorem, we use $p$ and $-\alpha$ to denote the order of regular variation of $V$ and $v$, respectively, where $p \in(-\infty, 0] \cup(1, \infty)$ and $\alpha \in(-\infty, 1) \cup(1,2]$ satisfy the one-to-one relationship

$$
\alpha=\frac{p-2}{p-1}
$$

with the additional convention that $p=\infty$ corresponds to $\alpha=1$. This notation is consistent with the notation used for the Tweedie family, where $\alpha \in(0,1) \cup(1,2]$ denotes the index of the stable distribution generating the Tweedie models with $p>2$ or $p \leqslant 0$.

A natural exponential family is said to be steep at $b$ if $b=\inf \Omega=\inf S$, with a similar definition at $\sup \Omega$, where $S$ denotes the support of the exponential family. If the family is steep at both end-points of $\Omega$, it is steep in the usual sense (cf. Barndorff-Nielsen 1978, p. 142).

As noted in connection with (1.3), the Lévy measures for members of an exponential family have the form $\mathrm{e}^{\theta x} v(\mathrm{~d} x)$. Hence, regular variation at zero is not affected by the value of $\theta$, whereas regular variation at infinity is. This is important to keep in mind when using the theorem. 
Theorem 2.4 (Tauber theorem for variance functions). Let $V$, with domain $\Omega$, be the variance function of a natural exponential family $\mathrm{P}$ generated by an infinitely divisible probability measure $w$ with Lévy-Khinchine representation

$$
k_{w}(s)=\int_{0}^{\infty}\left\{\mathrm{e}^{s x}-1-s \gamma(x)\right\} \nu(\mathrm{d} x) \quad \text { for } s \in \Theta_{w},
$$

where $v$ is a Lévy measure concentrated on $(0, \infty)$ and $\gamma(x)$ is a given centring function. Then there are four cases to consider:

Case (i): $p>1(\alpha<1)$. (a) Let $b=\inf \Omega$ be finite. Then $V$ is regularly varying at $b^{+}$ with exponent $p>1$, if and only if $v$ is regularly varying at zero with exponent $-\alpha$, where $\alpha<1$. (b) Let $\Theta_{w}=(-\infty, 0]$ and let $v$ be of type 0 or 1 . Then $\mathrm{P}$ is steep at zero and $V$ is regularly varying at infinity with exponent $p>1$ if and only if $v$ is regularly varying at infinity with exponent $-\alpha$, where $\alpha<1$.

Case (ii): $p<0(1<\alpha<2)$. (a) $V$ is regularly varying at $-\infty$ with exponent $p<0$ if and only if $v$ is regularly varying at zero with exponent $-\alpha$, where $1<\alpha<2$. (b) Let $v$ be of type 2 and let $\lim _{s \rightarrow 0} \tau(s)=0$. Then $V$ is regularly varying at $0^{-}$with exponent $p<0$, if and only if $v$ is regularly varying at $\infty$ with exponent $-\alpha$, where $1<\alpha<2$.

Case (iii): $p=0(\alpha=2)$. (a) $V$ is slowly varying at $-\infty$ if and only if $v$ is regularly varying at zero with exponent -2 . (b) Let $v$ be of type 2 and let $\lim _{s \rightarrow 0} \tau(s)=0$. Then $V$ is bounded regularly varying at $0^{-}$with exponent zero if and only if $v$ is bounded regularly varying at $\infty$ with exponent -2 .

Case (iv): $p=\infty(\alpha=1)$. (a) $V$ is bounded regularly varying at $-\infty$ with exponent $-\infty$ if and only if $v$ is bounded regularly varying at 0 with exponent -1 . (b) Let $v$ be of type 2. Then $V$ is bounded regularly varying at $\infty$ with exponent $\infty$ if and only if $v$ is bounded regularly varying at $\infty$ with exponent -1 .

In all cases, $V$ is bounded regularly varying if and only if $v$ is bounded regularly varying, under the hypotheses stated.

The theorem is proved in Section 4. A number of examples of regularly varying variance functions may be found in Jørgensen et al. (1994). The following example illustrates case (i) (a) of the theorem.

Example 2.5. Let $w$ be the infinitely divisible measure defined by

$$
w(\mathrm{~d} x)=\delta_{0}(\mathrm{~d} x)+1_{(0, \infty)}(x) \mathrm{d} x,
$$

where $\delta_{0}$ denotes the Dirac delta measure in 0 (cf. Letac 1992, p. 15). By Theorem 6.2 of Letac (1992, p. 12) the measure $\rho \in M(\mathbb{R})$ given by

$$
\rho(\mathrm{d} x)=x\left(1-\mathrm{e}^{-x}\right) 1_{(0, \infty)}(x) \mathrm{d} x
$$

is such that

$$
k_{w}^{\prime \prime}(\theta)=\int_{-\infty}^{\infty} \exp (\theta x) \rho(\mathrm{d} x) .
$$

If $\theta_{0}$ is in $\Theta_{w}$, then the Lévy measure of 


$$
w_{0}(\mathrm{~d} x)=\exp \left\{\theta_{0} x-k_{w}\left(\theta_{0}\right)\right\} w(\mathrm{~d} x)
$$

is

$$
v_{0}(\mathrm{~d} x)=x^{-1} \exp \left(\theta_{0} x\right)\left(1-\mathrm{e}^{-x}\right) 1_{(0, \infty)}(x) \mathrm{d} x .
$$

The measure $v_{0}$ is regularly varying at 0 with exponent 1 , corresponding to $\alpha=-1$. The natural exponential family generated by the measure $w_{0}$ has variance function $V(\mu)=$ $\mu\left(\mu^{2}+4 \mu\right)^{1 / 2}$. This function is regularly varying at 0 with exponent $p=1.5$.

Remark 2.6. Theorem 4.2 of Jørgensen et al. (1994) provides, with the appropriate reformulation in terms of regular variation, an easy corollary to the Tauber theorem for variance functions. Thus, if the measure $w$ generating the exponential dispersion model $\operatorname{ED}\left(\mu, \sigma^{2}\right)$ is infinitely divisible with Lévy-Khinchine representation (2.1), then under the appropiate conditions of the Tauber theorem for variance functions, bounded regular variation of the Lévy measure implies bounded regular variation of the variance function, and hence convergence of one of the forms (22) or (2) of Jørgensen et al. (1994).

The Tauber theorem for variance functions is limited to the case where the LévyKhinchine representation does not have a Gaussian component. The following example illustrates convergence to the normal distribution when such a component is present.

Example 2.7. Consider a measure $w_{c}$ with Lévy-Khinchine representation

$$
k_{w_{c}}(s)=c s^{2}+\int_{0}^{\infty}\left\{\mathrm{e}^{s x}-1-s \gamma(x)\right\} v(\mathrm{~d} x) \quad \text { for } s \in \Theta_{w}
$$

with Gaussian component $c s^{2}$. Let us suppose that the support of $w_{0}$ is contained in $[0, \infty)$, that $\Omega_{0}=(0, \infty)$ and that $\Theta_{0}=(-\infty, 0)$, where subscripts refer to $c$. Straightforward analysis shows that when $c>0$, we have that $\Omega_{c}=\mathbb{R}$ and

$$
V_{c}(\mu) \sim c \text { as } \mu \rightarrow-\infty, \quad V_{c}(\mu) \sim c+V_{0}(\mu) \text { as } \mu \rightarrow \infty .
$$

This shows that the distribution is locally normal at $-\infty$ (in the sense of Jørgensen et al. 1994), whereas the local behaviour at infinity depends on the relative orders of $c$ and $V_{0}$. This result should be compared with Example 5.1 of Jørgensen et al. (1994), the half-normal distribution, whose variance function is asymptotically constant at infinity, but where our result does not apply because the distribution is not infinitely divisible.

\section{Some results on regular variation}

This section contains some results regarding regular variation needed for the proof of Theorem 2.4. 
Proposition 3.1. Let $v$ be a Lévy measure of regular variation at zero with exponent $\beta-2$, $\beta>0$. Then the following statements hold:

(a) If $\beta>2, v$ is of type 0 .

(b) If $1<\beta<2, v$ is of type 1 .

(c) If $0<\beta<1, v$ is of type 2 .

(d) If $v$ is bounded regularly varying at 0 with exponent $\beta=1(\beta=2)$, then $v$ is of type 2 (type 1).

In the cases $\beta=1$ and $\beta=2$ it is not possible to state anything about the type of the measure based on regular variation of $v$ alone, as the following example illustrates.

Example 3.2. For a fixed $0<a<1$, we consider the Lévy measures $v_{k}$ on $(0, \infty)$ given by

$$
v_{k}(\mathrm{~d} x)=\frac{1_{(0, \alpha)}(x)}{x \log ^{k} x} \mathrm{~d} x,
$$

where $k \in \mathbb{N}$. Straightforward arguments show that $v_{k}$ is regularly varying in 0 with exponent 0 , corresponding to $\beta=2$. But $v_{k}$ is of type 0 if $k \geqslant 2$ and of type 1 if $k=1$. Similarly, the Lévy measures $x^{-1} v_{k}(\mathrm{~d} x)$ are regularly varying with exponent -1 in zero for all $k$, corresponding to $\beta=1$. But for $k=1$ the measure is of type 2 , and for $k>1$ of type 1 .

Proof of Proposition 3.1(a). Let $x>0$. To show that $v$ is of type 0 , we have to show that

$$
\int_{0}^{x} v(\mathrm{~d} t)<\infty
$$

First, note that

$$
\frac{x^{2}}{4} \int_{x / 2}^{x} v(\mathrm{~d} t) \leqslant \int_{x / 2}^{x} t^{2} v(\mathrm{~d} t) \leqslant \int_{0}^{x} t^{2} v(\mathrm{~d} t) .
$$

Since $v$ is regularly varying at zero with exponent $\beta-2$, it follows that

$$
\int_{0}^{x} t^{2} v(\mathrm{~d} t)=x^{\beta} L(x)
$$

where $L$ is slowly varying at zero. Hence, from (3.1) and (3.2) we obtain, for any $x>0$,

$$
\int_{x / 2}^{x} v(\mathrm{~d} x) \leqslant 4 x^{\beta-2} L(x)
$$

Now observe that

$$
\int_{0}^{x} v(\mathrm{~d} t)=\sum_{j=0}^{\infty} \int_{2^{-j-1} x}^{2^{-j} x} v(\mathrm{~d} t)
$$

So, from (3.3), 


$$
\int_{0}^{x} v(\mathrm{~d} t) \leqslant 4 x^{\beta-2} \sum_{j=0}^{\infty} 2^{-j(\beta-2)} L\left(2^{-j} x\right)
$$

Now, since $L$ is slowly varying at 0 , it follows that for all $\varepsilon>0$, there exists a $\sigma=\sigma(\varepsilon)$ such that

$$
1-\varepsilon \leqslant \frac{L\left(2^{-k-1} x\right)}{L\left(2^{-k+1} x\right)} \leqslant 1+\varepsilon
$$

for $0<2^{-k-1} x<\sigma$ and $k \geqslant-1$. Since

$$
L\left(2^{-j} x\right)=L(x) \frac{L\left(2^{-1} x\right)}{L(x)} \cdot \frac{L\left(2^{-2} x\right)}{L\left(2^{-1} x\right)} \ldots \frac{L\left(2^{-j} x\right)}{L\left(2^{-j-1} x\right)},
$$

we find

$$
L\left(2^{-j} x\right) \leqslant L(x)(1+\varepsilon)^{j} \leqslant L(x) \mathrm{e}^{j},
$$

for $\varepsilon$ small enough. Hence, for $0<x<\sigma$, we obtain from (3.4) and (3.5),

$$
\int_{0}^{x} v(\mathrm{~d} t) \leqslant 4 x^{\beta-2} L(x) \sum_{j=0}^{\infty} \mathrm{e}^{-j\{(\beta-2) \log 2-\varepsilon\}} .
$$

By assumption $\beta>2$, so we may choose $\varepsilon$ such that $(\beta-2) \log 2>\varepsilon$. Defining

$$
c=\sum_{j=0}^{\infty} \mathrm{e}^{-j\{(\beta-2) \log 2-\varepsilon\}},
$$

we obtain for $0<x<\sigma$,

$$
\int_{0}^{x} v(\mathrm{~d} t) \leqslant 4 c x^{\beta-2} L(x)<\infty
$$

which shows that $v$ is of type 0 .

Similar reasoning can be used to prove the remaining three cases of Proposition 3.1.

Proposition 3.3. Let $v$ be a Lévy measure that is bounded regularly varying at infinity with exponent $\beta-2, \beta>0$. Then the integral

$$
\int_{1}^{\infty} x v(\mathrm{~d} t)
$$

is convergent for $0<\beta<1$ and divergent for $\beta \geqslant 1$.

Proof. Let $0<\beta<1$. Then we obtain

$$
\int_{1}^{\infty} x v(\mathrm{~d} x) \leqslant \sum_{j=0}^{\infty} 2^{-j} \int_{2^{j}}^{2^{j+1}} x^{2} v(x) \leqslant \sum_{j=0}^{\infty} 2^{-j} \int_{0}^{2^{j+1}} x^{2} v(\mathrm{~d} x) .
$$


Since $v$ is bounded regularly varying at infinity with exponent $\beta-2$, it follows that

$$
\int_{0}^{2^{j+1}} x^{2} v(\mathrm{~d} x)=2^{\beta(j+1)} L\left(2^{j+1}\right),
$$

and hence,

$$
\int_{1}^{\infty} x v(\mathrm{~d} x) \leqslant 2^{\beta} \sum_{j=0}^{\infty} 2^{-j(1-\beta)} L\left(2^{j+1}\right)<\infty .
$$

Now consider $\beta \geqslant 1$. Then for $k>0$,

$$
\int_{1}^{\infty} x v(\mathrm{~d} x) \geqslant \sum_{j=k}^{\infty} 2^{-j-1} \int_{2^{j}}^{2^{j+1}} x^{2} v(\mathrm{~d} x)
$$

and

$$
\int_{2^{j}}^{2^{j+1}} x^{2} v(\mathrm{~d} x)=2^{(j+1) \beta} L\left(2^{j+1}\right)-2^{j \beta} L\left(2^{j}\right) .
$$

From (3.6) and (3.7) we obtain

$$
\int_{1}^{\infty} x v(\mathrm{~d} x) \geqslant \frac{1}{2} \sum_{j=k}^{\infty} 2^{j(\beta-1)}\left\{2^{\beta} L\left(2^{j+1}\right)-L\left(2^{j}\right)\right\} .
$$

Since $0<L(\infty)<\infty$, we may choose $\varepsilon>0$ such that

$$
a=L(\infty)\left\{(1-\varepsilon) 2^{\beta}-1-\varepsilon\right\}>0 .
$$

Thus,

$$
\int_{1}^{\infty} x v(\mathrm{~d} x) \geqslant \frac{a}{2} \sum_{j=k_{0}}^{\infty} 2^{j(\beta-1)}=\infty,
$$

provided $k_{0} \in \mathbb{N}$ is such that

$$
(1-\varepsilon) L(\infty) \leqslant L\left(2^{j}\right) \leqslant(1+\varepsilon) L(\infty) \quad \forall j \geqslant k_{0} .
$$

This completes the proof.

\section{Proof of the Tauber theorem for variance functions}

We now prove case (i) (a) of Theorem 2.4. The proofs of the remaining cases are fairly similar to this case. Note that Propositions 3.1 and 3.3 are not needed in this case, but are used in the proof of other cases. A complete proof is given in Jørgensen and Martínez (1993). 
Proof of case (i) (a) of Theorem 2.4. We first assume that $v$ is regularly varying at zero with exponent $-\alpha$, with $\alpha<1$. It is easy to see that we can assume that the measure $w$ has LévyKhinchine representation given by

$$
k_{w}(s)=\int_{0}^{\infty}\left(\mathrm{e}^{s x}-1\right) v(\mathrm{~d} x)
$$

We now show that $V$ is regularly varying at 0 with exponent $p>1$. We define $\tau_{-}:-\Theta_{w} \rightarrow \Omega$ by

$$
\tau_{-}(s)=\tau(-s)=k_{w}^{\prime}(-s) .
$$

By the monotone convergence theorem, we obtain that

$$
\tau_{-}(s) \rightarrow 0 \quad \text { as } s \rightarrow \infty .
$$

On the other hand, from (4.1) we obtain that

$$
-\tau_{-}^{\prime}(s)=\int_{0}^{\infty} \mathrm{e}^{-s x} x^{2} v(\mathrm{~d} x), \quad s \in-\Theta_{w},
$$

which is defined on $(0, \infty)$, allowing us to use the Tauber theorem.

By assumption, $v$ is regularly varying at 0 with exponent $-\alpha$, with $\alpha<1$, so from (4.3) we find that $-\tau_{-}^{\prime}$ is regularly varying at $\infty$ with exponent $\alpha-2<-1$. Then $\tau_{-}$is regularly varying at $\infty$ with exponent $\alpha-1$, because, for given $s>0$, using (4.2) and L'Hôpital's rule, we find

$$
\lim _{t \rightarrow \infty} \frac{\tau_{-}(s t)}{\tau_{-}(t)}=s \lim _{t \rightarrow \infty} \frac{\tau_{-}^{\prime}(s t)}{\tau_{-}^{\prime}(t)}=s^{\alpha-1},
$$

where the last equality of (4.4) follows because $\tau_{-}^{\prime}$ is regularly varying at $\infty$ with exponent $\alpha-2$. Now $\tau_{-}$is positive in $(0, \infty)$ and decreasing, so using $(4.2), \tau_{-}^{-1}$ is regularly varying at 0 with exponent $(\alpha-1)^{-1}$ (de Haan 1975, p. 22).

Now define the functions

$$
f(\psi)=-\tau_{-}^{\prime}\left(\frac{1}{\psi}\right), \quad g(\mu)=\frac{1}{\tau_{-}^{-1}(\mu)} .
$$

Then $f$ is regularly varying at 0 with exponent $2-\alpha$ and $g$ is regularly varying at 0 with exponent $(1-\alpha)^{-1}$. Using that $g(\mu) \rightarrow 0$ as $\mu \rightarrow 0$, it follows that $f\{g(\mu)\}=-\tau_{-}^{\prime}\left(\tau_{-}^{-1}(\mu)\right)$ $=V(\mu)$ is regularly varying at 0 with exponent $p=(\alpha-2) /(\alpha-1)$, where $p>1$.

Assume now that $V$ is regularly varying at 0 with exponent $p$, with $p>1$. This implies that $1 / V$ is regularly varying at 0 with exponent $-p$. Using the fact that

$$
\frac{\partial}{\partial \mu} \tau_{-}^{-1}(\mu)=\frac{1}{-V(\mu)}
$$

together with L'Hôpital's rule, we fine that $\tau_{-}^{-1}$ is regularly varying at zero with exponent $-p+1$. Since $\tau_{-}^{-1}(\mu) \rightarrow \infty$ as $\mu \rightarrow 0$, we find that $\tau_{-}$is regularly varying at infinity with exponent $(-p+1)^{-1}$. 
Observe now that $-\tau_{-}^{\prime}(s)=V\left\{\tau_{-}(s)\right\}$, that is, the composition of the functions $V(1 / \cdot)$ and $1 / \tau_{-}$, which are both regularly varying at $\infty$ with exponents $-p$ and $(p-1)^{-1}$, respectively. Thus $-\tau_{-}^{\prime}$ is regularly varying at $\infty$ with exponent $p /(1-p)$. Hence, from (4.3) and the Tauber theorem it follows that $v$ is regularly varying at zero with exponent $-\alpha=(2-p) /(p-1)$, where $\alpha<1$. This completes the proof.

\section{Acknowledgement}

The work on this paper was supported by NATO collaborative research grant 921347 .

\section{References}

Bar-Lev, S.K. (1987) Discussion of B. Jørgensen: 'Exponential Dispersion Models'. J. Roy. Statist. Soc. Ser B, 49, 153-154.

Bar-Lev, S.K. and Enis, P. (1986) Reproducibility and natural exponential families with power variance functions. Ann. Statist., 14, 1507-1522.

Bar-Lev, S.K. and Bshouty, D. (1989) Rational variance functions. Ann. Statist., 17, 741-748.

Bar-Lev, S.K. and Bshouty, D. (1990) A class of infinitely divisible variance functions with application to the polynomial case. Statist. Probab. Lett., 10, 377-379.

Bar-Lev, S.K., Bshouty, D. and Enis, P. (1991a) Variance functions with meromorphic means. Ann. Probab., 19, 1349-1366.

Bar-Lev, S.K., Bshouty, D. and Enis, P. (1991b) Infinitely divisible variance functions: Survey of recent developments. Paper presented at the 48th Session of the International Statistical Institute, Cairo.

Barndorff-Nielsen, O.E. (1978) Information and Exponential Families. Chichester: Wiley.

Bingham, N.H., Goldie, C.M. and Teugels, J.L. (1987) Regular Variation. Cambridge: Cambridge University Press.

De Haan, L. (1975) On Regular Variation and Its Application to the Weak Convergence of Sample Extremes, Mathematical Centre Tracts 32. Amsterdam: Mathematisch Centrum.

Feller, W. (1971) An Introduction to Probability Theory and Its Applications, Vol. 2 (2nd edn). New York: Wiley.

Jørgensen, B. (1986) Some properties of exponential dispersion models. Scand. J. Statist., 13, 187198.

Jørgensen, B. (1987) Exponential dispersion models (with discussion). J. Roy. Statist. Soc. Ser B, 49, $127-162$.

Jørgensen, B. (1992) The Theory of Exponential Dispersion Models and Analysis of Deviance (2nd edn), Monograf. Mat. 51. Rio de Janeiro: IMPA.

Jørgensen, B. and Martínez, J.R. (1993) Tauber theory for infinitely divisible variance functions. Technical Report No. 133, Department of Statistics, University of British Columbia.

Jørgensen, B. and Martinez, J.R. and Tsao, M. (1994) Asymptotic behaviour of the variance function. Scand. J. Statist., 21, 223-243.

Letac, G. (1987) Discussion of B. Jørgensen: 'Exponential Dispersion Models'. J. Roy. Statist. Soc. Ser. B., 49, 154.

Letac, G. (1992) Lectures on Natural Exponential Families and Their Variance Functions, Monograf. Mat. 50. Rio de Janeiro: IMPA. 
Letac, G. and Mora, M. (1990) Natural real exponential families with cubic variances. Ann. Statist. 18, $1-37$.

McCullagh, P. and Nelder, J.A. (1989) Generalized Linear Models (2nd edn). London: Chapman \& Hall.

Mora, M. (1986) Classification des fonctions variance cubiques des familles exponentielles sur $\mathbb{R}$. C.R. Acad. Sci. Paris I, 302, 587-590.

Mora, M. (1990) La convergence des fonctions variances des familles exponentielles naturelles. Ann. Fac. Sci. Toulouse Math. (5), 11, 105-120.

Morris, C.N. (1982) Natural exponential families with quadratic variance functions. Ann. Statist., 10, $65-80$.

Tweedie, M.C.K. (1947) Functions of a statistical variate with given means, with special reference to Laplacian distributions. Proc. Cambridge Phil. Soc., 49, 41-49.

Wedderburn, R.W.M. (1974) Quasi-likelihood functions, generalized linear models and the GaussNewton method. Biometrika, 61, 435-447.

Received February 1995 and revised October 1996. 